PROCEEDINGS OF THE AMERICAN MATHEMATICAL SOCIETY

Volume 124, Number 5, May 1996

\title{
ROTATIONAL SYMMETRY OF THE HERMITE PROJECTION OPERATORS
}

\author{
E. KOCHNEFF
}

(Communicated by J. Marshall Ash)

\begin{abstract}
We calculate an integral formula for the Hermite projection operators. We give some applications of our formula. We also give a short proof of a recent theorem of Thangavelu
\end{abstract}

\section{INTRODUCTION}

In a recent paper ([T], Thangavelu) it was shown that if $P(x)$ is a solid spherical harmonic of degree $m$ and if $f \in L^{2}\left(R^{n}\right)$ is of the form $f(x)=f_{0}(|x|) P(x)$, then its projection $\mathcal{P}_{k} f(x)$ onto the space of Hermite functions of degree $k$ is a Laguerre function multiplied by $P(x)$.

More specifically, let $L_{k}^{\delta}$ denote the Laguerre polynomials of type $\delta$. Define

$$
R_{k}^{\delta}\left(f_{0}\right)=2 \frac{k !}{\Gamma(k+\delta+1)} \int_{0}^{\infty} f_{0}(r) L_{k}^{\delta}\left(r^{2}\right) e^{-r^{2} / 2} r^{2 \delta+1} d r .
$$

If $f(x)=f_{0}(|x|) P(x) \in L^{2}\left(R^{n}\right)$, then one has $\mathcal{P}_{2 k+m} f(x)=F_{k}(|x|) P(x)$ where

$$
F_{k}(r)=R_{k}^{\delta}\left(f_{0}\right) L_{k}^{\delta}\left(r^{2}\right) e^{-r^{2} / 2}
$$

with $\delta=\frac{n}{2}+m-1$. For other values of $j, \mathcal{P}_{j} f=0$. The proof is based on generating functions.

Since Hermite functions are eigenfunctions of the Fourier transform and Laguerre functions are eigenfunctions of the Fourier-Bessel (Hankel) transform, Thangavelu's formula is closely related to the Hecke-Bochner identity which says that if $f \in$ $L^{1} \cap L^{2}\left(R^{n}\right)$ such that $f(x)=f_{0}(|x|) P(x)$, then $\hat{f}(x)=F_{0}(|x|) P(x)$ where

$$
\begin{gathered}
\hat{f}(x)=\int_{R^{n}} f(y) e^{-i x \cdot y} d y, \\
F_{0}(r)=(2 \pi)^{n / 2} i^{-m} r^{-(n / 2+m-1)} \int_{0}^{\infty} f_{0}(s) J_{n / 2+m-1}(r s) s^{n / 2+m} d s
\end{gathered}
$$

and $J_{\alpha}$ is the Bessel function of order $\alpha$. For a proof of this identity see [SW].

One of the important properties of the Fourier transform is its symmetry with respect to rotations. Thangavelu's formula (1.2) suggests the same should be true for the projection operators $\mathcal{P}_{k}$. Our main result in this paper is an integral formula

Received by the editors November 4, 1994.

1991 Mathematics Subject Classification. Primary 33C50, 42C10; Secondary 33C55.

Key words and phrases. Fourier transform, spherical harmonics, Hermite and Laguerre polynomials. 
for the kernels of the projection operators which shows that the projection operators commute with rotations. We will apply our formula to give a simple proof of Thangavelu's formula in the radial case and to give an expression for Laguerre polynomials in terms of Hermite polynomials. We will also give a short proof of Thangavelu's formula which only uses $L^{2}$ theory for the Hermite and Laguerre polynomials and the homogeneity of the spherical harmonics.

\section{Definitions}

Define $\mathcal{H}_{m}$ to be the space of all $f \in L^{2}\left(R^{n}\right)$ of the form $f_{0}(|x|) P(x)$ where $P(x)$ is a (solid) spherical harmonic of degree $m$, i.e., $P(x)$ is a homogeneous harmonic polynomial of degree $m$. These spaces provide a direct sum decomposition of $L^{2}\left(R^{n}\right)$ invariant under the Fourier transform; see [SW].

The Hermite polynomials orthogonal with respect to the weight $e^{-x^{2}} d x$ on $R$ are given for $k=0,1, \ldots$ by

$$
H_{k}(x)=(-1)^{k} \frac{d^{k}}{d x^{k}}\left(e^{-x^{2}}\right) e^{x^{2}}=\sum_{j=0}^{\left[\frac{k}{2}\right]} \frac{(-1)^{j} k !}{j !(k-2 j) !}(2 x)^{k-2 j} .
$$

They satisfy the orthogonality relation

$$
\int_{R} H_{j}(x) H_{k}(x) e^{-x^{2}} d x=2^{k} k ! \sqrt{\pi} \delta_{j, k} .
$$

The Hermite functions $H_{k}(x) e^{-x^{2} / 2}$ are eigenfunctions of the Fourier transform:

$$
H_{k}(x) e^{-x^{2} / 2}=\frac{i^{k}}{\sqrt{2 \pi}} \int_{-\infty}^{\infty} H_{k}(y) e^{-y^{2} / 2-i x y} d y, \quad k=0,1, \ldots
$$

The Hermite polynomials on $R^{n}$ are defined for multi-indices $\mu=\left(\mu_{1}, \ldots, \mu_{n}\right)$, $\mu_{i}=0,1, \ldots$, as products of one-dimensional Hermite polynomials:

$$
H_{\mu}(x)=H_{\mu_{1}}\left(x_{1}\right) H_{\mu_{2}}\left(x_{2}\right) \cdots H_{\mu_{n}}\left(x_{n}\right), \quad x=\left(x_{1}, \ldots, x_{n}\right) .
$$

Note that if $|\mu|=\mu_{1}+\mu_{2}+\cdots+\mu_{n}$ is even, then all terms in $H_{\mu}$ are of even degree. The Hermite functions $H_{\mu}(x) e^{-|x|^{2} / 2}$ are orthogonal on $R^{n}$ :

$$
\int_{R^{n}} H_{\mu}(x) H_{\nu}(x) e^{-|x|^{2}} d x=2^{|\mu|} \mu ! \pi^{n / 2} \delta_{\mu, \nu}
$$

where

$$
\mu !=\mu_{1} ! \mu_{2} ! \cdots \mu_{n} !
$$

They are eigenfunctions of the Fourier transform in $R^{n}$.

A function $f \in L^{2}\left(R^{n}\right)$ can be expanded in series of Hermite functions using the formula

$$
f(x) \sim \sum_{k=0}^{\infty} \mathcal{P}_{k} f(x)
$$

where $\mathcal{P}_{k} f$ are the Hermite projection operators

$$
\mathcal{P}_{k} f(x)=\sum_{|\mu|=k} \hat{f}(\mu) H_{\mu}(x) e^{-|x|^{2} / 2},
$$




$$
\hat{f}(\mu)=\frac{1}{2^{k} \mu ! \pi^{n / 2}} \int_{R^{n}} f(y) H_{\mu}(y) e^{-|y|^{2} / 2} d y .
$$

In other words,

$$
\mathcal{P}_{k} f(x)=\int_{R^{n}} f(y) \Phi_{k}(x, y) d y
$$

where

$$
\Phi_{k}(x, y)=\frac{1}{2^{k} \pi^{n / 2}} \sum_{|\mu|=k} \frac{H_{\mu}(x) H_{\mu}(y)}{\mu !} e^{-\left(|x|^{2}+|y|^{2}\right) / 2} .
$$

For many interesting theorems concerning expansions in terms of these polynomials, see $[\mathrm{T} 2]$.

For $\alpha>-1$ the Laguerre polynomials $\left\{L_{k}^{\alpha}(x)\right\}$ of type $\alpha$ are defined by the formula

$$
e^{-x} x^{\alpha} L_{k}^{\alpha}(x)=\frac{1}{k !} \frac{d^{k}}{d x^{k}}\left(e^{-x} x^{k+\alpha}\right)=\sum_{j=0}^{k} \frac{\Gamma(k+\alpha+1)}{\Gamma(j+\alpha+1)} \frac{(-x)^{j}}{j !(k-j) !} .
$$

The Laguerre polynomials satisfy the orthogonality

$$
\int_{0}^{\infty} L_{k}^{\alpha}(x) L_{j}^{\alpha}(x) e^{-x} x^{\alpha} d x=\frac{\Gamma(k+\alpha+1)}{k !} \delta_{k, j} .
$$

Note that this implies that $|x|^{m} L_{k}^{\frac{n-2}{2}+m}\left(|x|^{2}\right) e^{-|x|^{2} / 2}, k=0,1, \ldots$, are orthogonal on $R^{n}$ for each $m$.

The set consisting of the functions $L_{k}^{\alpha}(2 x) x^{\alpha / 2} e^{-x}$ are eigenfunctions of the Hankel transform (see e.g. $[\mathrm{Sz}])$ :

$$
L_{k}^{\alpha}(2 x) x^{\alpha / 2} e^{-x}=\int_{0}^{\infty} J_{\alpha}(2 \sqrt{x y}) L_{k}^{\alpha}(2 y) y^{\alpha / 2} e^{-y} d y, \quad k=0,1, \ldots .
$$

This implies by the Hecke-Bochner identity that the functions

$$
L_{k}^{\frac{n-2}{2}+m}\left(|x|^{2}\right) P(x) e^{-|x|^{2} / 2}, \quad k=0,1, \ldots,
$$

which span the space $\mathcal{H}_{m}$ are eigenfunctions of the Fourier transform in $R^{n}$.

\section{A formula FOR the PROJECTION OPERATOR}

Lemma 3.1. Let $u=\left(u_{1}, u_{2}, \ldots, u_{n}\right)$ be a unit vector in $R^{n}$, and let $x \in R^{n}$. Then for $k=0,1, \ldots$

$$
H_{k}(x \cdot u)=\sum_{|\mu|=k}\left(\begin{array}{l}
k \\
\mu
\end{array}\right) H_{\mu}(x) u^{\mu}
$$

where $u^{\mu}=u_{1}^{\mu_{1}} u_{2}^{\mu_{2}} \ldots u_{n}^{\mu_{n}}$ and $\left(\begin{array}{l}k \\ \mu\end{array}\right)$ is the multinomial index $k ! / \mu !$.

Proof. For $n=2$ this formula is well known:

$$
H_{k}(x \cos \theta+y \sin \theta)=\sum_{j=0}^{k}\left(\begin{array}{l}
k \\
j
\end{array}\right) H_{j}(x) H_{k-j}(y) \cos ^{j} \theta \sin ^{k-j} \theta .
$$

The more general formula is proved inductively. Let $u$ be a unit vector in $R^{n+1}$. Then we can write

$$
u=\left(\cos \theta,(\sin \theta) v_{1},(\sin \theta) v_{2}, \ldots,(\sin \theta) v_{n}\right)
$$


where $v=\left(v_{1}, v_{2}, \ldots, v_{n}\right)$ is a unit vector in $R^{n}$. Let $x=\left(x_{1}, x_{2}, \ldots, x_{n+1}\right) \in R^{n+1}$. Let $y=\left(x_{2}, x_{3}, \ldots, x_{n+1}\right) \in R^{n}$. Then

$$
x \cdot u=x_{1} \cos \theta+y \cdot v \sin \theta
$$

so that we have

$$
\begin{aligned}
H_{k}(x \cdot u) & =\sum_{j=0}^{k}\left(\begin{array}{l}
k \\
j
\end{array}\right) H_{j}\left(x_{1}\right) H_{k-j}(y \cdot v) \cos ^{j} \theta \sin ^{k-j} \theta \\
& =\sum_{j=0}^{k}\left(\begin{array}{l}
k \\
j
\end{array}\right) H_{j}\left(x_{1}\right) \sum_{|\nu|=k-j}\left(\begin{array}{c}
k-j \\
\nu
\end{array}\right) H_{\nu}(y) v^{\nu} \cos ^{j} \theta \sin ^{k-j} \theta \\
& =\sum_{|\mu|=k}\left(\begin{array}{l}
k \\
\mu
\end{array}\right) H_{\mu}(x) u^{\mu} .
\end{aligned}
$$

Theorem 3.2. For $k=0,1, \ldots$

$$
\Phi_{k}(x, y)=\frac{(-i)^{k} e^{\left(|x|^{2}-|y|^{2}\right) / 2}}{\pi^{n} k !} \int_{R^{n}}|s|^{k} H_{k}\left(y \cdot \frac{s}{|s|}\right) e^{-|s|^{2}+2 i s \cdot x} d s .
$$

Proof. It is well known (see e.g. [Sz]) that

$$
H_{k}(x)=\frac{2^{k}(-i)^{k} e^{x^{2}}}{\sqrt{\pi}} \int_{-\infty}^{\infty} s^{n} e^{-s^{2}+2 i s x} d s, \quad k=0,1, \ldots
$$

It easily follows for $|\mu|=k$ that

$$
H_{\mu}(x)=\frac{2^{k}(-i)^{k} e^{|x|^{2}}}{\pi^{n / 2}} \int_{R^{n}} s^{\mu} e^{-|s|^{2}+2 i s \cdot x} d s .
$$

Therefore we have

$$
\begin{aligned}
\sum_{|\mu|=k}\left(\begin{array}{c}
k \\
\mu
\end{array}\right) H_{\mu}(x) H_{\mu}(y) & =\sum_{|\mu|=k}\left(\begin{array}{c}
k \\
\mu
\end{array}\right)\left(\frac{2^{k}(-i)^{k} e^{|x|^{2}}}{\pi^{n / 2}} \int_{R^{n}} s^{\mu} e^{-|s|^{2}+2 i s \cdot x} d s\right) H_{\mu}(y) \\
& =\frac{2^{k}(-i)^{k} e^{|x|^{2}}}{\pi^{n / 2}} \int_{R^{n}}\left\{\sum_{|\mu|=k}\left(\begin{array}{c}
k \\
\mu
\end{array}\right) H_{\mu}(y) s^{\mu}\right\} e^{-|s|^{2}+2 i s \cdot x} d s \\
& =\frac{2^{k}(-i)^{k} e^{|x|^{2}}}{\pi^{n / 2}} \int_{R^{n}}|s|^{k} H_{k}\left(y \cdot \frac{s}{|s|}\right) e^{-|s|^{2}+2 i s \cdot x} d s .
\end{aligned}
$$

Finally since

$$
\Phi_{k}(x, y)=\frac{e^{-\left(|x|^{2}+|y|^{2}\right) / 2}}{2^{k} k ! \pi^{n / 2}} \sum_{|\mu|=k}\left(\begin{array}{l}
k \\
\mu
\end{array}\right) H_{\mu}(x) H_{\mu}(y),
$$

we obtain the result.

Note that for $n=1(3.3)$ reduces to $H_{k}(x) H_{k}(y) e^{-\left(x^{2}+y^{2}\right) / 2} / 2^{k} \sqrt{\pi} k$ ! as expected. 
Using $H_{2 k}(0)=(-1)^{k}(2 k) ! / k$ ! and the Hecke-Bochner formula we obtain

$$
\begin{aligned}
\Phi_{2 k}(x, 0) & =\frac{e^{|x|^{2} / 2}}{\pi^{n} k !} \int_{R^{n}} e^{2 i s \cdot x}|s|^{2 k} e^{-|s|^{2}} d s \\
& =\frac{2 e^{|x|^{2} / 2}}{\pi^{n / 2} k !|x|^{\frac{n-2}{2}}} \int_{0}^{\infty} J_{\frac{n-2}{2}}(2 r|x|) r^{2 k+\frac{n}{2}} e^{-r^{2}} d r \\
& =\frac{e^{-|x|^{2} / 2}}{\pi^{n / 2}} L_{k}^{\frac{n-2}{2}}\left(|x|^{2}\right)
\end{aligned}
$$

where we have used

$$
L_{m}^{\nu}(x)=\frac{e^{x} x^{-\nu / 2}}{m !} \int_{0}^{\infty} s^{m+\nu / 2} J_{\nu}(2 \sqrt{x s}) e^{-s} d s,
$$

see e.g. [Sz]. On the other hand,

$$
\Phi_{2 k}(x, 0)=\frac{e^{-|x|^{2} / 2}}{2^{2 k} \pi^{n / 2}} \sum_{|\mu|=2 k} \frac{H_{\mu}(0)}{\mu !} H_{\mu}(x) .
$$

Therefore we obtain

Corollary 3.3. For $k=0,1, \ldots$

$$
L_{k}^{\frac{n-2}{2}}\left(|x|^{2}\right)=\frac{1}{2^{2 k}} \sum_{|\mu|=2 k} \frac{H_{\mu}(0)}{\mu !} H_{\mu}(x) .
$$

Note that $\Phi_{2 k+1}(x, 0)=0$ for all $x$ and $k=0,1, \ldots$

Theorem 3.4. Let $R_{\rho} f(x)=f(\rho x)$ where $\rho$ is a rotation. Then for $k=0,1, \ldots$

$$
\mathcal{P}_{k} R_{\rho} f(x)=R_{\rho} \mathcal{P}_{k} f(x) .
$$

Proof. This follows from the observation that

$$
\Phi_{k}(\rho x, \rho y)=\Phi_{k}(x, y), \quad k=0,1, \ldots,
$$

which follows easily from (3.3).

\section{A proof of Thangavelu's formula in the Radial Case}

Theorem 3.2 leads to a proof of Thangavelu's formula in the radial case.

One can evaluate the integral

$$
\int_{\Sigma_{n-1}} H_{2 k}\left(x \cdot u^{\prime}\right) d u^{\prime}
$$

by first integrating over the parallel

$$
L_{\theta}=\left\{u^{\prime} \in \Sigma_{n-1}: x^{\prime} \cdot u^{\prime}=\cos \theta\right\}
$$


and then integrating over $\theta, 0 \leq \theta \leq \pi$. Then since $\omega_{n-2} \sin ^{n-2} \theta$ is the measure of $L_{\theta}$, we have

$$
\begin{aligned}
\int_{\Sigma_{n-1}} H_{2 k}\left(x \cdot u^{\prime}\right) d u^{\prime} & =\omega_{n-2} \int_{0}^{\pi} H_{2 k}(r \cos \theta) \sin ^{n-2} \theta d \theta \\
& =\omega_{n-2} \int_{-1}^{1} H_{2 k}(r t)\left(1-t^{2}\right)^{\frac{n-3}{2}} d t \\
& =\frac{2 \pi^{n / 2}(-1)^{k}(2 k) !}{\Gamma\left(k+\frac{n}{2}\right)} L_{k}^{\frac{n-2}{2}}\left(r^{2}\right)
\end{aligned}
$$

where in the last step we have used the well-known formula of Uspenski (see [Sz]):

$$
L_{k}^{\alpha}(x)=\frac{(-1)^{k} \pi^{-1 / 2} \Gamma(k+\alpha+1)}{\Gamma\left(\alpha+\frac{1}{2}\right)(2 k) !} \int_{-1}^{1} H_{2 k}(\sqrt{x} t)\left(1-t^{2}\right)^{\alpha-\frac{1}{2}} d t, \quad \alpha>-\frac{1}{2} .
$$

Letting $|y|=r$ and $|x|=\gamma$ we obtain

$$
\begin{aligned}
\int_{\Sigma_{n-1}} \Phi_{2 k}(x, y) d y^{\prime}= & \frac{(-1)^{k} e^{\left(r^{2}-\gamma^{2}\right) / 2}}{\pi^{n}(2 k) !} \\
& \cdot \int_{R^{n}}|s|^{2 k}\left\{\int_{\Sigma_{n-1}} H_{2 k}\left(r y^{\prime} \cdot s^{\prime}\right) d y^{\prime}\right\} e^{-|s|^{2}+2 i s \cdot x} d s \\
= & \frac{2 k !}{\Gamma\left(k+\frac{n}{2}\right)} L_{k}^{\frac{n-2}{2}}\left(r^{2}\right) L_{k}^{\frac{n-2}{2}}\left(\gamma^{2}\right) e^{-\left(r^{2}+\gamma^{2}\right) / 2} .
\end{aligned}
$$

Therefore if $f(x)=f_{0}(|x|) \in L^{2}\left(R^{n}\right)$ we obtain Thangavelu's formula:

$$
\begin{aligned}
\mathcal{P}_{2 k} f(x) & =\int_{R^{n}} f(y) \Phi_{2 k}(x, y) d y \\
& =\int_{0}^{\infty} f_{0}(r)\left\{\int_{\Sigma_{n-1}} \Phi_{2 k}\left(x, r y^{\prime}\right) d y^{\prime}\right\} r^{n-1} d r \\
& =R_{k}^{\frac{n-2}{2}}\left(f_{0}\right) L_{k}^{\frac{n-2}{2}}\left(\gamma^{2}\right) e^{-\gamma^{2} / 2} .
\end{aligned}
$$

It is easy to see that $\mathcal{P}_{2 k+1} f(x)=0$.

Uspensky's formula can be generalized to give a proof of Thangavalu's formula in the general case. However, the constants become very unwieldy. At any rate, the generalization is given in Section 6 .

\section{A short Proof of Thangavelu's formula}

Lemma 5.1. If $|\nu|<m$ or $|\nu|-m$ is odd and $P(x)$ is a spherical harmonic of degree $m$, then

$$
\int_{\Sigma_{n-1}}\left(x^{\prime}\right)^{\nu} P\left(x^{\prime}\right) d x^{\prime}=0
$$

Proof. Let $|\nu|=k$ so that $x^{\nu}$ is a homogeneous polynomial of degree $k$. Thus we can write

$$
x^{\nu}=P_{0}(x)+|x|^{2} P_{1}(x)+\cdots+|x|^{2 l} P_{l}(x)
$$


where $P_{j}$ is a spherical harmonic of degree $k-2 j, j=0,1, \ldots, l$; see $[\mathrm{SW}]$. Therefore, since spherical harmonics are orthogonal on the unit sphere, we have the result.

Let $H_{\mu, m}(x)$ be a "cut" Hermite polynomial. That is, all terms of degree $\leq m-1$ are dropped. Then Lemma 5.1 shows that

$$
\int_{\Sigma_{n-1}} H_{\mu}\left(r x^{\prime}\right) P\left(x^{\prime}\right) d x^{\prime}=\int_{\Sigma_{n-1}} H_{\mu, m}\left(r x^{\prime}\right) P\left(x^{\prime}\right) d x^{\prime} .
$$

Theorem 5.2. Let $P(x)$ be a spherical harmonic of degree $m$. Define for $k=$ $0,1, \ldots$

$$
F_{k}(x)=L_{k}^{\frac{n-2}{2}+m}\left(|x|^{2}\right) P(x) e^{-|x|^{2} / 2}
$$

Then

$$
\mathcal{P}_{j} F_{k}(x)= \begin{cases}F_{k}(x) & \text { if } j=2 k+m, \\ 0 & \text { otherwise. }\end{cases}
$$

Proof. (For $m=0$ this follows from Corollary 3.3.) Note that each $F_{k}(x)$ can be written as a linear combination of Hermite functions of degree $\leq 2 k+m$. Therefore the theorem will follow if we show that $\widehat{F}_{k}(\mu)=0,|\mu|<2 k+m$. Consider the function $\psi_{\mu}(r)$ defined for $r>0$ by

$$
\psi_{\mu}\left(r^{2}\right)=\frac{1}{r^{m}} \int_{\Sigma_{n-1}} H_{\mu}\left(r y^{\prime}\right) P_{m}\left(y^{\prime}\right) d y^{\prime} .
$$

By Lemma 5.1, if $|\mu|+m$ is odd or $|\mu|<m$, then $\psi_{\mu}\left(r^{2}\right)=0$. If $|\mu|=2 j+m$, $j=0,1, \ldots$, then

$$
\psi_{\mu}\left(r^{2}\right)=\frac{1}{r^{m}} \int_{\Sigma_{n-1}} H_{\mu, m}\left(r y^{\prime}\right) P_{m}\left(y^{\prime}\right) d y^{\prime} .
$$

In this case $\psi_{\mu}(r)$ is a polynomial of degree $j$ so that if $j<k$ we have

$$
\int_{0}^{\infty} \psi_{\mu}(r) L_{k}^{\frac{n-2}{2}+m}(r) e^{-r} r^{\frac{n-2}{2}+m} d r=0
$$

Therefore, if $|\mu|<2 k+m$ by switching to polar coordinates we have

$$
\begin{aligned}
\widehat{F}_{k}(\mu) & =\frac{1}{2^{k} \mu ! \pi^{n / 2}} \int_{R^{n}} L_{k}^{\frac{n-2}{2}+m}\left(|y|^{2}\right) P_{m}(y) H_{\mu}(y) e^{-|y|^{2}} d y \\
& =\frac{1}{2^{k} \mu ! \pi^{n / 2}} \int_{0}^{\infty} \psi_{\mu}\left(r^{2}\right) L_{k}^{\frac{n-2}{2}+m}\left(r^{2}\right) e^{-r^{2}} r^{n+2 m-1} d r \\
& =0 .
\end{aligned}
$$

This completes the proof.

Theorem 5.3 (Thangavelu's formula). Define

$$
R_{k}^{\delta}\left(f_{0}\right)=2 \frac{k !}{\Gamma(k+\delta+1)} \int_{0}^{\infty} f_{0}(r) L_{k}^{\delta}\left(r^{2}\right) e^{-r^{2} / 2} r^{2 \delta+1} d r .
$$

Then if $f(x)=f_{0}(|x|) P(x) \in L^{2}\left(R^{n}\right)$, then one has $\mathcal{P}_{2 k+m} f(x)=F_{k}(|x|) P(x)$ 
where

$$
F_{k}(r)=R_{k}^{\delta}\left(f_{0}\right) L_{k}^{\delta}\left(r^{2}\right) e^{-r^{2} / 2}
$$

with $\delta=\frac{n}{2}+m-1$. For other values of $j, \mathcal{P}_{j} f=0$.

Proof. Since $f \in L^{2}\left(R^{n}\right)$, we have $f_{0}(r) \in L^{2}\left((0, \infty) ; r^{2 \delta+1} d r\right)$. Thus

$$
f_{0}(r)=\sum_{i=0}^{\infty} R_{i}^{\delta}\left(f_{0}\right) L_{i}^{\delta}\left(r^{2}\right) e^{-r^{2} / 2}
$$

converging in the $L^{2}\left((0, \infty) ; r^{2 \delta+1} d r\right)$ norm. This implies that

$$
f(x)=\sum_{i=0}^{\infty} R_{i}^{\delta}\left(f_{0}\right) L_{i}^{\delta}\left(|x|^{2}\right) e^{-|x|^{2} / 2} P(x)
$$

converging in $L^{2}\left(R^{n}\right)$. But $\mathcal{P}_{j}$ are bounded operators in $L^{2}\left(R^{n}\right)$; therefore the result follows from Theorem 5.2.

\section{A generalization of Uspensky's Formula}

Let $P_{m}^{\lambda}(t), m=0,1, \ldots$, denote the ultraspherical polynomials of order $\lambda$. Then since for $u, x^{\prime} \in \Sigma_{n-1}$

$$
Z_{u}^{(m)}\left(x^{\prime}\right)=c_{m, n} P_{m}^{\frac{n-2}{2}+m}\left(u \cdot x^{\prime}\right)
$$

where $Z_{u}^{(m)}\left(x^{\prime}\right)$ denotes the zonal harmonic of degree $m$ and pole $u$ (see [SW]), Thangavelu's formula could be proved from the following generalization of Uspensky's formula.

Theorem 6.1. For $k, m=0,1, \ldots, \lambda>-1$

$$
\int_{-1}^{1} H_{2 k+m}(r t) P_{m}^{\lambda}(t)\left(1-t^{2}\right)^{\lambda-\frac{1}{2}} d t=c_{m, k} r^{m} L_{k}^{\lambda+m}\left(r^{2}\right)
$$

where

$$
\begin{gathered}
c_{m, k}=\frac{H_{2 k+m}^{(m)}(0) \lambda_{m}}{m ! \gamma_{m} L_{k}^{\lambda+m}(0)}, \\
\gamma_{m}=2^{m} \frac{\Gamma(m+\lambda)}{m ! \Gamma(\lambda)}
\end{gathered}
$$

is the coefficient of $t^{m}$ in $P_{m}^{\lambda}(t)$ and

$$
\lambda_{m}=\int_{-1}^{1}\left[P_{m}^{\lambda}(t)\right]^{2}\left(1-t^{2}\right)^{\lambda-\frac{1}{2}} d t
$$


Proof. We use the Rodriguez formulas; see e.g. [Sz]. For simplicity, let $c_{m, k}$ below be a constant which may change with each usage. We have

$$
\begin{aligned}
\int_{-1}^{1} & H_{2 k+m}(r t) P_{m}^{\lambda}(t)\left(1-t^{2}\right)^{\lambda-\frac{1}{2}} d t \\
& =c_{m, k} \int_{-1}^{1} H_{2 k+m}(r t)\left(\left(\frac{d}{d t}\right)^{m}\left(1-t^{2}\right)^{\lambda+m-\frac{1}{2}}\right) d t \\
& =c_{m, k} \int_{-1}^{1}\left(\left(\frac{d}{d t}\right)^{m} H_{2 k+m}(r t)\right)\left(1-t^{2}\right)^{\lambda+m-\frac{1}{2}} d t \\
& =c_{m, k} r^{m} \int_{-1}^{1} H_{2 k}(r t)\left(1-t^{2}\right)^{\lambda+m-\frac{1}{2}} d t \\
& =c_{m, k} r^{m} L_{k}^{\lambda+m}\left(r^{2}\right) .
\end{aligned}
$$

Let $H_{j, m}(x)$ denote the $j$ th Hermite polynomial with all terms of degree $\leq m-1$ dropped. Then

$$
\int_{-1}^{1} H_{2 k+m}(r t) P_{m}^{\lambda}(t)\left(1-t^{2}\right)^{\lambda-\frac{1}{2}} d t=\int_{-1}^{1} H_{2 k+m, m}(r t) P_{m}^{\lambda}(t)\left(1-t^{2}\right)^{\lambda-\frac{1}{2}} d t
$$

so that $c_{m, k}$ can be calculated by dividing both sides in (6.6) by $r^{m}$ and setting $r=0$.

\section{REFERENCES}

[Sz] G. Szegö, Orthogonal polynomials, Amer. Math. Soc. Colloq. Publ., vol. 23, Amer. Math. Soc., Providence, RI, 1975. MR 51:8724

[T] S. Thangavelu, Hermite expansions on $R^{n}$ for radial functions, Proc. Amer. Math. Soc. 118 (1993), 1097-1102. MR 93j:42016

[T2] Lectures on Hermite and Laguerre expansions, Princeton Univ. Press, Princeton, NJ, 1993. MR 94i:42001

[SW] E. Stein and G. Weiss, Introduction to Fourier analysis on Euclidean spaces, Princeton Univ. Press, Princeton, NJ, 1981,

Department of Mathematics, Eastern Washington University, Cheney, Washington 99004

E-mail address: ekochneff@ewu.edu 